\title{
Modification de la sensibilité au lindane d'Asellus aquaticus L. en fonction de la variation de facteurs biotiques (poids et métabolisme) et abiotiques (concentration de l'insecticide et température) \\ Sensitivity of Asellus aquaticus L. (Crustacea, Isopoda) to lindane in relation to biotic (size and metabolism) and abiotic factors (concentration of insecticide and temperature)
}

\section{S. Le Bras}

Volume 3, numéro 2, 1990

URI : https://id.erudit.org/iderudit/705070ar

DOI : https://doi.org/10.7202/705070ar

Aller au sommaire du numéro

Éditeur(s)

Université du Québec - INRS-Eau, Terre et Environnement (INRS-ETE)

\section{ISSN}

0992-7158 (imprimé)

1718-8598 (numérique)

\section{Découvrir la revue}

\section{Citer cet article}

Le Bras, S. (1990). Modification de la sensibilité au lindane d'Asellus aquaticus L. en fonction de la variation de facteurs biotiques (poids et métabolisme) et abiotiques (concentration de l'insecticide et température). Revue des sciences de l'eau / Journal of Water Science, 3(2), 183-193. https://doi.org/10.7202/705070ar
Résumé de l'article

La sensibilité d'Asellus aquaticus L. (Crustacea, Isopada) à un insecticide, le lindane, est étudiée. Pour chaque concentration de lindane (1, 2, 4 et 8 mg.L $\mathrm{L}^{-1}$ ) la mortalité est appréciée en fonction du poids des individus ou de leur métabolisme. La sensibilité à $4 \mathrm{mg} . \mathrm{L}^{-1}$ de lindane à des températures différentes $\left(10\right.$ et $\left.20^{\circ} \mathrm{C}\right)$ a été également envisagée. La sensibilité des aselles s'accroit avec la concentration de lindane, la température et le métabolisme. Il en est de même aux concentrations sublétales quand le poids augmente. 


\title{
Modification de la sensibilité au lindane d'Asellus aquaticus $L$. en fonction de la variation de facteurs biotiques (poids et métabolisme) et abiotiques (concentration de l'insecticide et température)
}

\author{
Sensitivity of Asellus aquaticus L. (Crustacea, Isopoda) \\ to lindane in relation to biotic (size and metabolism) and \\ abiotic factors (concentration of insecticide and temperature)
}

\section{Suzette LE BRAS1}

RÉSUMÉ

La sensibilité d'Asellus aquaticus L. (Crustacea, Isopoda) à un insecticide, le lindane, est étudiée. Pour chaque concentration de lindane $\left(1,2,4\right.$ et $\left.8 \mathrm{mg} . \mathrm{L}^{-1}\right)$ la mortalité est appréciée en fonction du poids des individus ou de leur mélabolisme. La sensibilité à $4 \mathrm{mg} \cdot \mathrm{L}^{-1}$ de lindane à des températures différentes (10 et $20^{\circ} \mathrm{C}$ ) a été également envisagée. La sensibilité des aselles s'accroit avec la concentration de lindane, la température et le métabolisme. If en est de méme aux concentrations sublétales quand le poids augmente.

Mots clés : Asellus aquaticus, lindane, senslbilté, ecophase.

SUMMARY

The sensitivity of Asellus aquaticus L. (Crustacea, Isoposa) to the insecticide lindane was studied in relation to its developmental stage (size) and metabolism.Atter tests of acute toxicity ( $48 \mathrm{~h}$ ) with 1, 2, 4 or $8 \mathrm{mg} \cdot \mathrm{L}^{-1}$ of lindane, the percentage of mortality increased with the concentration. In further tests using sublethal concentrations, 1 or $2 \mathrm{mg} . \mathrm{L}^{-1}$, the sensitivity increased with the weight of the organisms. A linear regression of mortality (probit of \%) of Aset Ius was correlated with the mean concentration $(M)$ of lindane and the weight (W) of Asellus for these two concentrations. It is possible to relate the percentage of mortality to the metabolic rate observed at the beginning of the experiment. The larger Asellus and/or those with a higher metabolic rate were the most sensitive. An increase in temperature which modified the metabolism, increased the sensitibity of this invertebrate. The percentage of mortality were respectively 1.35 and 26.64 at 10 and $20^{\circ} \mathrm{C}$. The interest of these results lies in the evaluation of the risks of pollution. In this type of study, it is very important to consider the biotic factors (size, metabolism) because the concentration of insecticide is generally close to a sublethal concentrations. Indeed biolic

1. C.N.R.S. - URA 20, Laboratoire de zoologie et d'écologie, Bat. 442, Université de Paris-Sud, 91405 Orsay Cedex, France. 
factors that modify the sensitivity are the main elements responsible for the it tensity of the harm caused. The abiotic factor (temperature) that changes the physiology of invertebrate is also a significant factor.

Key-words: Asellus aquaticus, Indane, sens/tivity, developmental stage.

\section{INTRODUCTION}

Les résultats des tests de toxicité obtenus par les toxicologistes dans des expériences in vitro ne peuvent être que rarement extrapolés au domaine écotoxicologique, et toujours avec beaucoup de réserves.

En effet, dans le but de pouvoir comparer diverses substances, dérivés d'un même élément chimique ou de formulations variées, les toxicologistes de l'environnement doivent opérer dans des conditions expérimentales standardisées : échantillons homogènes (sexe, âge, état physiologique, taille, etc.), facteurs abiotiques précis (température, $\mathrm{pH}$, luminosité, etc.) (BUIKEMA et al., 1982). Or dans la nature tous ces facteurs interviennent simultanément et modifient considérablement l'action d'un pesticide et les conséquences de son emploi.

II serait donc d'un grand intérêt de pouvoir déterminer non pas seulement la toxicité d'un composé mais aussi le degré de sensibilité de la population sur laquelle il va agir, selon son état d'évolution et (ou) selon laction de différents facteurs abiotiques. L'idéal serait de trouver un indice de sensibilité tenant compte de tous ces facteurs et non pas seulement de la concentration du produit comme c'est le cas dans les déterminations des CL50 et des DL50.

Parmi les nombreux éléments pouvant modifier la toxicité d'un pesticide sur une espèce donnée, le stade de son développement (écophase) représente un paramètre de toute première importance.

Le but de cette étude a donc été de déterminer le degré de toxicité d'un pesticide, le lindane, sur un crustacé isopode : Asellus aquaticus L., en fonction de son poids et de son métabolisme, lesquels constituent en première approximation la meilleure façon de quantifier le stade de développement pour ce type d'organisme qui ne présente pas de stades morphologiques marqués.

La difficulté de cette étude réside dans le fait que poids et métabolisme sont étroitement liés. Pour dissocier ces deux facteurs nous avons été amené à faire varier la température, car chez les invertébrés le métabolisme dépend en premier lieu de celle-ci.

Nous avons donc étudié :

1. La sensibilité au lindane à différentes concentrations en fonction du poids des individus.

2. La variation de la sensibilité en fonction de la température pour une concentration sublétale de lindane.

3. Les relations entre la sensibilité et le métabolisme initial. 


\section{MATÉRIEL ET MÉTHODES}

L'organisme de référence choisi pour cette étude, $A$. aquaticus $L$. est un arthropode limnique qui, en tant que ressource alimentaire pour de nombreux poissons et par son régime alimentaire (détritivore), se situe à la base des chaînes trophiques et présente de ce fait une importance écologique certaine d'autant plus qu'il a tendance à pulluler dans les eaux présentant une charge en matières organiques fermentescibles (HYNES, 1960).

Pour notre étude, cette espèce est récoltée dans le milieu naturel (étang de la ville d'Orsay) et est acclimatée aux conditions du laboratoire à la température ambiante, dans de l'eau oxygénée. Elle est alimentée avec des feuilles d'érable récoltées aux environs du lieu de prélèvement.

\section{Sensibilité en fonction du poids}

L'intoxication est réalisée à $18^{\circ} \mathrm{C}$, en lumière artificielle (tubes au néon) avec une photopériode de $12 \mathrm{~h}$. Les aselles sont pesées individuellement et réparties en classe d'intervalle de $4 \mathrm{mg}$. Dix individus d'une même classe sont déposés dans des cristallisoirs de $250 \mathrm{ml}$. Pour éviter leur agrégation on place une feuille d'aluminium $(7 \times 7 \mathrm{~cm})$ froissée qui sert à la fois de support et d'abri, les aselles présentant un phototactisme négatif. Après $48 \mathrm{~h}$ les animaux vivants sont décomptés. Le pourcentage de mortalité est évalué puis corrigé selon la formule d'Abbott (FINNEY, 1971) en fonction de la mortalité observée chez les témoins.

Les concentrations de lindane (produit fourni par La Quinoléine - pur à $99 \%$ ) testées ont été les suivantes : 1, 2, 4 et $8 \mu \mathrm{g} . \mathrm{L}^{-1}$. Pour chacune d'entre elles et pour chaque classe de poids trois replicats ont été effectués pour épreuve toxicologique.

L'interprétation des résultats a été réalisée en appliquant la formule de BLISS (1936) (1) développée par ANDERSON et WEBER (1975).

$$
Y=a+b \log M W^{h}
$$

dans laquelle:

$y=$ probit du pourcentage de morts

$M=$ concentration moyenne du toxique

$W=$ poids de l'animal

$\mathrm{h}=$ exposant à définir selon les espèces de manière à obtenir le meilleur coefficient de corrélation possible

Dans notre expérimentation (test sur $48 \mathrm{~h}$ ) nous avons considéré que $M$ était égal à la concentration de départ. En effet d'après les travaux de THYBAUD (1987), il n'y a pas de dégradation du lindane dans l'eau pendant cette période de temps. Par ailleurs le meilleur coefficient de corrélation a été obtenu pour $\mathrm{h}=1$. 


\section{Sensibilité en fonction de la température}

Cette étude a été réalisée à la concentration sublétale de $4 \mu \mathrm{g} \cdot \mathrm{L}^{-1}$. Le pourcentage de mortalité est apprécié selon le protocole d'intoxication indiqué précédemment, seule la température a été modifiée. Nous avons opéré à 10 et $20^{\circ} \mathrm{C}$. Pour chaque température le résultat représente la moyenne obtenue à partir de 30 répétitions, soit 6 tests pour chacune des 5 classes de poids considérées.

\section{Sensibilité en fonction du métabolisme}

L'intoxication par $4 \mu \mathrm{g} \cdot \mathrm{L}^{-1}$ de findane a été réalisée dans des aquariums de verre $(15 \times 20 \times 20 \mathrm{~cm})$, contenant 2 । d'eau, $8 \mathrm{ml}$ de solution acétonique de lindane à la concentration désirée, une feuille d'aluminium servant à la fois de support et d'abri aux aselles, en absence d'aération et avec 80 aselles'. Pour chaque température 5 répétitions ont été réalisées.

Après $48 \mathrm{~h}$, les aselles sont récupérées et le pourcentage de morts est noté. Les survivants sont placés dans un milieu dépourvu de tindane puis leur métabolisme est mesuré par le taux de consommation d'oxygène. Ce dernier facteur est évalué par la méthode polarographique utilisant une électrode de Clark avec un radiometer type E 5046. Dans ce but, les aselles sont conservées à la température d'étude dans des tubes $(5 \mathrm{ml}$ environ) contenant de l'eau saturée en oxygène et fermés hermétiquement. Nous mettons deux individus de même taille par tube. Après une incubation de 2 à $3 \mathrm{~h}$ on mesure la pression $d^{\prime} \mathrm{O}_{2}$ dans l'eau. La consommation d'oxygène est exprimée en $\mu \mathrm{g} \cdot \mathrm{L}^{-1} \cdot \mathrm{min}^{-1}$.

\section{RÉSULTATS}

\section{Sensibilité en fonction du poids}

L'application de la formule (1) nous a permis de calculer l'équation d'une droite de régression et le coefficient de corrélation pour deux des concentrations étudiées (tableau 1).

Ces derniers comparés à ceux lus dans la table publiée par FiSCHER et YATES (1963) indiquent que la relation entre la mortalité et le poids de l'animal est significative au seuil 0,05 et 0,001 respectivement pour 1 et $2 \mu \mathrm{g} . \mathrm{L}^{-1}$ de lindane. Par contre à 4 et $8 \mu \mathrm{g} \cdot \mathrm{L}^{-1}$ le pourcentage de mortalité semble indépendant du poids des animaux (relation non significative).

La comparaison par un test $t$ des pentes des droites obtenues pour 1 et 2 $\mu \mathrm{g} . \mathrm{L}^{-1}$ (fig. 1) indique que celles-ci sont semblables; seules les ordonnées à l'origine diffèrent.

1. Ces différents paramètres (volume d'eau, de solution, nombre d'individus etc.) ont élé choisis de manière à conserver les mèmes proportions que dans les tests de toxicité. 
D'après ces résultats il apparaît que la toxicité du lindane varie en fonction du poids de l'animal des concentrations sublétales voisines de la CL10 $\left(1,73 \pm 0,28 \mu \mathrm{g} . \mathrm{L}^{-1}\right.$ intervalle de confiance à $\left.95 \%\right)$ THYBAUD et al. (1987).

Tableau 1 Equation des droites de régression de la mortalité $Y$ (probit du \% morts) d'Asellus aquaticus $L$. en fonction de la concentration moyenne $(M)$ de lindane et du poids de l'aselle $(W)$, après une intoxication aiguë (48 h) par 1 ou $2 \mu \mathrm{g} . \mathrm{L}^{-1}$ de lindane.

Table 1 Linear regression of mortality (probit of \%) of Asellus aquaticus $L$., correlation with mean concentration of lindane $(M)$ and weight of Asellus (W) after acute intoxication (48 h) with 1 or $2 \mu \mathrm{g} \cdot \mathrm{L}^{-1}$ of lindane.

\section{Lindane $1 \mu \mathrm{g} . \mathrm{L}^{-1}$}

Equation de la droite : $Y=2,8692-2,1838 \log (\mathrm{M} / \mathrm{W})$

$R=-0,87$

analyse de variance :

\begin{tabular}{|lllll|}
\hline Origine de la varlation & Sonme des carrés & dl & Carré moven & F \\
\hline Régression & 2,61 & 1 & 2,61 & 9,54 \\
Résiduelle & 0,82 & 3 & 0,27 & \\
Total & 3,43 & 4 & & \\
\hline
\end{tabular}

\section{Llndane $2 \mu \mathrm{g} \cdot \mathrm{L}^{-1}$}

Equation de la droite : $Y=3,6854-1,9725 \log (M / W)$

$R=-0,97$

analyse de variance :

\begin{tabular}{|lllll|}
\hline Orliglne de la varlation & Somme des carrés & dl & Carré moyen & F \\
\hline Régression & 8,06 & 1 & 8,06 & 110,68 \\
Résiduelte & 0,51 & 7 & 0,07 & \\
Total & 8,57 & 8 & & \\
\hline
\end{tabular}

D'après l'équation de la droite (tableau 1) on peut estimer que pour une concentration de lindane de $1 \mu \mathrm{g} . \mathrm{L}^{-1}$ par exemple le pourcentage de mortalité pourra varier de $2,3 \%$ à $80 \%$ suivant que les aselles péseront entre 1 et $20 \mathrm{mg}$, intervalle de poids qui correspond à la majorité des aselles d'une population.

\section{Sensibilité en fonction de la température}

A $10^{\circ} \mathrm{C}$ nous constatons après correction d'Abbott une mortalité de $1,35 \%$ pour $4 \mu \mathrm{g} \cdot \mathrm{L}^{-1}$. La comparaison par un test ANOVA de la mortalité observée chez les témoins $(7,54 \%)$ et les intoxiqués $(8,79 \%)$ indique $F=$ 4,29 pour 60 et 1 DDL (tableau 2). La mortalité chez les intoxiqués n'est donc pas significativement différente de celle des témoins. 
A $20^{\circ} \mathrm{C}$ la mortalité observée chez les intoxiqués est après correction d'Abbott de $26,64 \%$. La comparaison de la mortalité chez les témoins $(13,21 \%)$ et les intoxiqués $(36,33 \%)$ indique que la mortalité chez ces derniers est significative au seuil 0,001 ( $F=19,96$ pour 1 et $56 \mathrm{DDL}$ ) (tableau 2).

Tableau 2 Taux de mortalité d'Asellus aquaticus L., témoins et intoxiqués par $4 \mu \mathrm{g} . \mathrm{L}^{-1}$ de lindane pendant $48 \mathrm{~h}$, à diftérentes températures.

Table 2 Mortality of Asellus aquaticus $L$. in control and after acute contamination $(48 \mathrm{~h})$ by $4 \mu \mathrm{g} \cdot \mathrm{L}^{-1}$ of lindane at different temperatures.

\begin{tabular}{|c|c|c|c|}
\hline Température & Témolns & Intoxlqués & Test ANOVA \\
\hline $10^{\circ} \mathrm{C}$ & $\begin{array}{l}n=29 \\
m=7,54 \\
s=9,3902\end{array}$ & $\begin{array}{l}n=33 \\
m=8,79 \\
s=10,8275\end{array}$ & $\begin{array}{l}F=\quad 4,29 \\
\text { pour } 60 \text { et } 1 \text { ddl } \\
F=252,2 \text { n.s. }\end{array}$ \\
\hline $20^{\circ} \mathrm{C}$ & $\begin{array}{l}n=28 \\
m=13,21 \\
s=20,0099\end{array}$ & $\begin{array}{l}n=30 \\
m=36,33 \\
s=19,3842\end{array}$ & $\begin{array}{l}F=19,96 \\
\text { pour i et } 56 \mathrm{ddl} \\
F=12 \text { s. d } 0,001\end{array}$ \\
\hline $\begin{array}{l}\text { Test } \\
\text { ANOVA }\end{array}$ & $\begin{array}{l}F=1,89 \\
\text { pour } 1 \text { el } 55 \text { ddf } \\
F=4 \\
\text { n.s. }\end{array}$ & $\begin{array}{l}F=41,86 \\
\text { pour } 1 \text { et } 61 \text { ddl } \\
F^{\prime}=11,97 \\
\text { s. à } 0,001\end{array}$ & \\
\hline
\end{tabular}

Ainsi nous constatons une différence de sensibilité en fonction de la température, à $10^{\circ} \mathrm{C}, 4 \mu \mathrm{g} . \mathrm{L}^{-1}$ de lindane ne provoque aucune mortalité alors qu'à $20^{\circ} \mathrm{C}$ elle est de l'ordre de $27 \%$.

Par ailleurs à cette température nous n'avons constaté, comme à $18^{\circ} \mathrm{C}$, aucune relation entre le taux de mortalité et le poids.

Ces résultats ne peuvent s'expliquer par des différences de taille puisque chaque expérience a été réalisée avec les mêmes classes de poids. Le lindane n'étant pas considéré comme thermo-labile, nous avons recherché si la différence de sensibilité observée entre les deux températures n'était pas liée à la modification physiologique de l'aselle en particulier à la variation de son métabolisme. En effet nous avons établi que le Q10 entre 10 et $20^{\circ} \mathrm{C}$ était de l'ordre de 2,29.

\section{Sensibilité en fonction du métabolisme}

Dans le tableau 3 nous avons noté la consommation d'oxygène enregistrée chez les témoins (colonne 2) et après intoxication (colonne 5) ainsi que la mortalité observée (colonnes 3 et 7) dans chacun des replicats réalisés. De plus, lintoxication pouvant modifier le métabolisme (LE BRAS, 1987), nous ne pouvons pas utiliser les valeurs de la consommation $\mathrm{d}^{\prime} \mathrm{O}_{2}$ des individus intoxiqués pour exprimer une éventuelle relation entre le métabolisme et le taux de mortalité. Pour palier à cet inconvénient nous avons considéré que le métabolisme des intoxiqués était le même au départ de l'expérimentation que celui des lots témoins correspondants (tous les individus provenaient du 
même stock). Ainsi à partir des équations de consommation d' $\mathrm{O}_{2}$ obtenues pour les témoins :

$$
\log d e \text { VO2 }=A \log d u \text { poids sec }+B
$$

et sachant que :

$$
\log \text { poids sec }=0,9926 \log \text { pds.frais }-0,6458
$$

nous avons pu estimer, en fonction de leur poids, la consommation $\mathrm{d}^{\prime} \mathrm{O}_{2}$ des intoxiqués au début de l'expérimentation (colonne 6).

Tableau 3 Métabolisme et mortalité observés dans les lots d'Asellus aquaticus $\mathrm{L}$. témoins et intoxiqués par $4 \mu \mathrm{g} . \mathrm{L}^{-1}$ de lindane pendant $48 \mathrm{~h}$ à 10 et $20^{\circ} \mathrm{C}$.

PF. Poids Frais (en $\mathrm{mg}$ ), $\mathrm{VO}_{2}$. consommation d' $\mathrm{O}_{2}$ (en $\left.\mu \mathrm{g} \cdot \mathrm{L}^{-1} \cdot \mathrm{min}^{-1}\right), \% \mathrm{M}$ et \% Mc. pourcentages de mortalité observés et corrigés, $\mathrm{VO}_{2}$.cal. consommation ${ }^{\prime} \mathrm{O}_{2}$ estimée à partir de celle des témoins.

Table 3 Mortality and metabolic rate of Asellus aquaticus $L$. control and after acute contamination $(48 \mathrm{~h})$ with $4 \mu \mathrm{g} \cdot \mathrm{L}^{-1}$ of lindane at 10 and $20^{\circ} \mathrm{C}$.

PF. wet weight $(\mathrm{mg}), \mathrm{VO}_{2}$ consumption of $\mathrm{O}_{2}\left(\mu g \cdot \mathrm{L}^{-1} \cdot \mathrm{min}^{-1}\right), \% \mathrm{M}$ and $\%$ Mc. percentage of mortality observed and corrected, $\mathrm{VO}_{2} . \mathrm{cal}$.

\begin{tabular}{|c|c|c|c|c|c|c|c|c|c|}
\hline \multicolumn{5}{|c|}{ Témolns } & \multicolumn{5}{|c|}{ Intoxiques } \\
\hline \multicolumn{10}{|l|}{$10^{\circ} \mathrm{C}$} \\
\hline & (1) & (2) & (3) & & (4) & (5) & (6) & (7) & (8) \\
\hline rêf. & $\mathrm{PF}$ & $\mathrm{VO}_{2}$ & $\% M$ & rếf. & PF & $\mathrm{VO}_{2}$ & $\mathrm{VO}_{2} \mathrm{cal}$. & $\% M$ & $\% M C$ \\
\hline MT101 & 4,14 & 0,0058 & 6,25 & ML4101 & 3,53 & 0,0077 & 0,0106 & 7,50 & 1,33 \\
\hline MT10 & 23,16 & 0,0049 & 5,00 & ML4102 & 2,83 & 0,0047 & 0,0084 & 6,25 & 1,32 \\
\hline MT103 & 3,81 & 0,0065 & 5,00 & ML4103 & 4,78 & 0,0057 & 0,0119 & 7,77 & 2,92 \\
\hline MT104 & 9,63 & 0,0160 & 0,00 & ML4104 & 9,62 & 0,0145 & 0,0229 & 3,75 & 3,75 \\
\hline MT105 & 7,08 & 0,0080 & 6,25 & ML4105 & 7,36 & 0,0126 & 0,0151 & 5,00 & 0 \\
\hline \multicolumn{10}{|l|}{$20^{\circ} \mathrm{C}$} \\
\hline MT201 & 7,76 & 0,0285 & 10,00 & ML4201 & 7,20 & 0,0244 & 0,0319 & 25,00 & 16,67 \\
\hline MT202 & 5,95 & 0,0187 & 7,50 & ML4202 & 6,72 & 0,0237 & 0,0325 & 50,00 & 45,95 \\
\hline MT203 & 5,70 & 0,0172 & 7,50 & ML4203 & 4,66 & 0,0159 & 0,0188 & 21,11 & 14,71 \\
\hline MT204 & 3,49 & 0,0140 & 5,00 & ML4204 & 3,47 & 0,0116 & 0,0200 & 8,89 & 4,09 \\
\hline MT205 & 3,61 & 0,0141 & 7,50 & $M L 4205$ & 4,70 & 0,0182 & 0,0252 & 21,11 & 14,71 \\
\hline
\end{tabular}
consumption of $\mathrm{O}_{2}$ calculated from that of the control.

Ainsi il nous a été possible de calculer la régression entre le $\mathrm{VO}_{2}$ calculé et le taux de mortalité chez les intoxiqués, corrigé par rapport à celui des témoins (tableau 4).

Le coefficient de corrélation $R$ obtenu $(0,7697)$ est supérieur à celui lu dans la table de FISCHER et YATES (1963) $\left(R^{\prime}=0,7646\right)$ au seuil 0,01 . La droite de régression du pourcentage de mortalité en fonction de la consommation d'O $_{2}$ initiale est tracée (fig.2).

Le pourcentage de mortalité est donc bien lié au niveau métabolique initial. 
II nous est donc possible d'affirmer que l'accroissement de sensibilité noté quand le poids et (ou) la température s'élèvent est on grande partie lié à l'évolution du métabolisme causée par la variation de ces deux facteurs.

Tableau 4 Equation de la droite de régression de la mortalité (\%) d'Asellus aquaticus $\mathrm{L}$. intoxiqué par $4 \mu \mathrm{g} \cdot \mathrm{L}^{-1}$ de lindane pendant $48 \mathrm{~h}$, en fonction de son métabolisme (consommation d'oxygène en $\mu \mathrm{g} \cdot \mathrm{L}^{-1} \cdot \mathrm{min}^{-1}$ ).

Table 4 Linear regression of mortality (\%) of Asellus aquaticus $L$., after acute contamination ( $48 \mathrm{~h}$ ) by $4 \mu \mathrm{g} . \mathrm{L}^{-1}$ of lindane correlation to metabolic rate (consumption of $\mathrm{O}_{2}$ in $\mu \mathrm{g} \cdot \mathrm{L}^{-1} \cdot \mathrm{min}^{-1}$ ).

Equation de la droite : $\%$ de mort $=1267,43$ cons. $O_{2}-14,4613$

$R=-0,7697$

Analyse de variance :

\begin{tabular}{|lllll|}
\hline Origine de la variation & Somme des carrés & Carré moyen & F \\
\hline Régression & 1038,62 & 1 & 1038,62 & 9,91 \\
Résidueite & 714,30 & 8 & 89,29 & \\
Total & 1752,92 & 9 & & \\
\hline
\end{tabular}

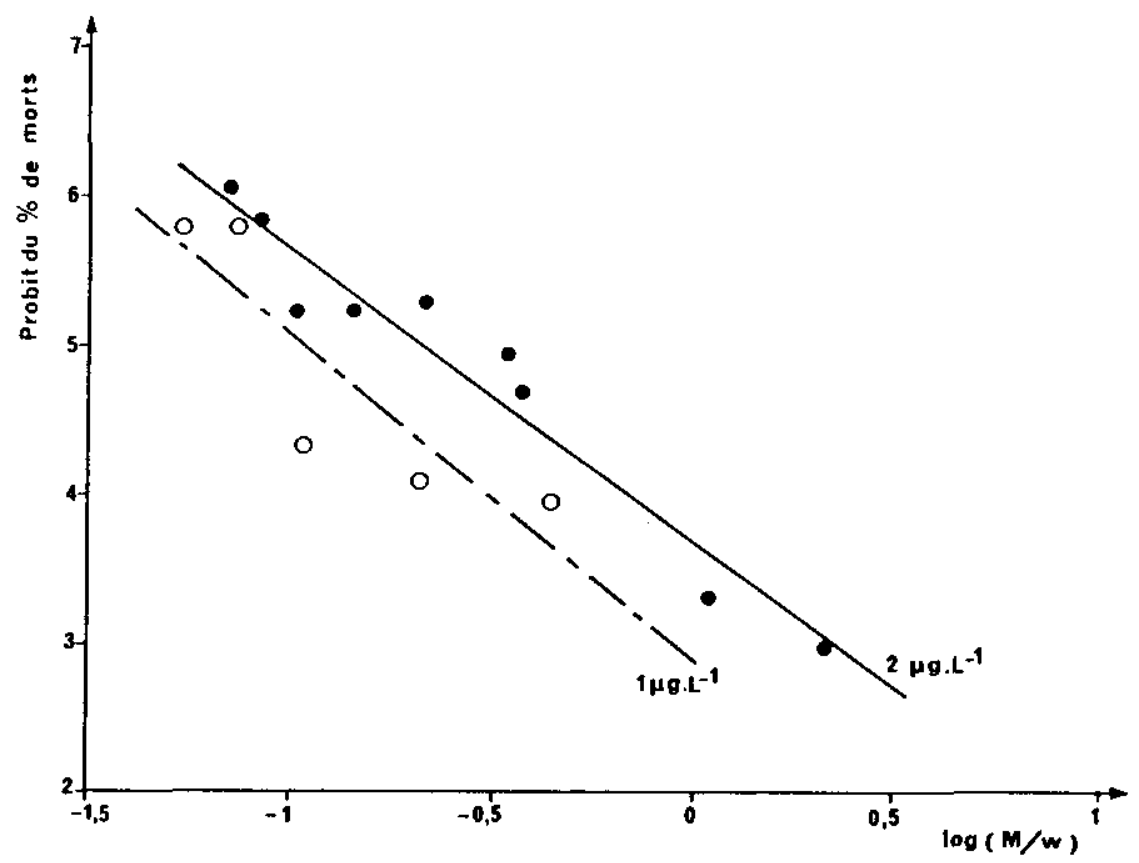

Figure 1 Variation du taux de mortalité chez Asellus aquaticus L. après intoxication par 1 et $2 \mu \mathrm{g} . \mathrm{L}^{-1}$ de lindane pendant $48 \mathrm{~h}$ en fonction du poids des aselles.

Variation of the mortality rate of Asellus aquaticus $L$. after acute contamination (during $48 \mathrm{~h}$ ) with 1 and $2 \mu \mathrm{g} \cdot \mathrm{L}^{-1}$ of lindane in relation to its weight. 


\section{DISCUSSION}

La modification de la sensibilité d'un organisme en fonction de son poids est très bien connue chez de nombreux poissons (ANDERSON et WEBER, 1975 ; PHILLIPS, 1980) et même chez des oiseaux (TUCKER et LEITZKE, 1979). D'une manière générale il semble que contrairement à ce que nous observons chez les aselles, les individus de petite taille soient plus sensibles que les gros. Ce phénomène peut trouver une explication dans le fait que, en règle générale, l'absorption étant un phénomène de surface, les petits individus ayant un rapport surface/masse supérieur aux individus de grande taille absorbent toute chose égale par ailleurs, une plus grande quantité d'insecticide par rapport à leur poids.

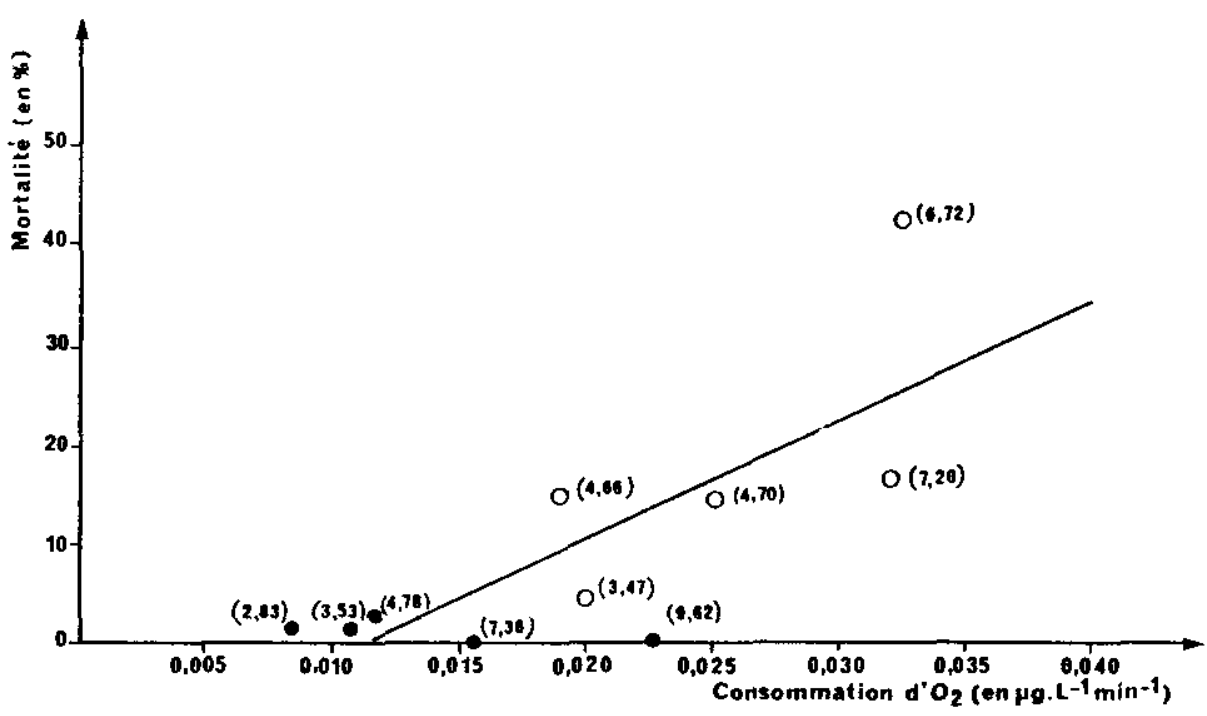

Figure 2 Taux de mortalité d'Asellus aquaticus $L$. en fonction du niveau métabolique après intoxication par $4 \mu \mathrm{g} \cdot \mathrm{L}^{-1}$ de lindane pendant $48 \mathrm{~h}$ à $10(\bullet)$ et $20^{\circ} \mathrm{C}(\mathrm{O})$ (entre parenthèse poids frais).

Mortality of Asellus aquaticus $L$. in relation to its metabolic rate, after acute contamination (during $48 \mathrm{~h}$ ) with $4 \mu \mathrm{g} \cdot \mathrm{L}^{-1}$ of lindane at $10^{\circ} \mathrm{C}(\mathrm{C})$ and $20^{\circ} \mathrm{C}(\mathrm{O})$ (wet weight, in brackets).

A notre connaissance peu de travaux ont été réalisés à ce sujet sur des invertébrés aquatiques. Cependant, STEPHENSON (1983), ne constate aucune modification de la sensibilité de Gammarus pulex (L.) en fonction de la taille, vis-à-vis du phénol, du cuivre, du lindane et du di-éthylhexyl phtalate (DEHP). Toutefois il faut noter que les concentrations utilisées pour cette étude sont de l'ordre de la CL50. Et en conséquence ces observations ne sont pas en contradiction avec ce que nous constatons chez Asellus.

Ces résultats suggèrent que l'accumulation plus importante de toxique chez les jeunes n'est pas le seul facteur intervenant dans les différences de 
sensibilité des diverses écophases d'invertébrés aquatiques. En particulier le métabolisme semble jouer un rôle important.

En effet nous venons de mettre en évidence que chez les aselles la sensibilité s'accroît avec la consommation d'oxygène.

Ainsi l'accroissement du métabolisme, dû à l'augmentation de poids, on sait en effet que celui-ci est fonction du poids $\left(R=a W^{b}, R\right.$ désigne le métabolisme et $W$ le poids) (LE BRAS, 1987), ou bien à une élévation de température, va augmenter la sensibilité des aselles.

Enfin une interprétation possible de ces résultats pourrait être la suivante : il semble que la quantité de lindane pénétrant dans les individus soit fonction à la fois du poids et (ou) du métabolisme, et de sa concentration dans le milieu (THYBAUD et LE BRAS, 1987). Dans te cas des faibles concentrations c'est la variation des paramètres biologiques qui va faire la différence d'un individus à lautre, alors que pour les concentrations élevées cette variation métabolique devient négligeable par rapport à la quantité de lindane présente dans le milieu.

Ainsi pourrait-on expliquer que dans le cas de faibles concentrations on ait observée une différence de sensibilité en fonction du poids et (ou) du métabolisme, alors que celle-ci disparaît aux concentrations plus élevées.

Le fait que la sensibilité des aselles varie en fonction du poids à des concentrations infralétales est particulièrement important dans la mesure où dans les phénomènes de contamination chimique du milieu par des résidus de pesticides, ce sont précisément à de faibles concentrations de pesticides que sont exposées les biocénoses aquatiques, aussi risquent-ils de provoquer un déséquilibre dans la population en faisant disparaître préférentiellement les individus les plus âgés. Nous venons de voir que $1 \mu \mathrm{g} \cdot \mathrm{L}^{-1}$ de lindane ferait disparaître $80 \%$ des individus de $10 \mathrm{mg}$ et seulement $2 \%$ des aselles de $1 \mathrm{mg}$.

Cet effet sélectif a, semble-t-il, été effectivement observé dans la nature chez des poissons à la suite d'intoxication dans un lac naturel par du toxaphène (insecticide organo-halogéné) (HEMPHILL, 1954, FUKANO et HOPPER, 1958 cités par PHILLIPS, 1980). Mais à l'inverse des aselles ce sont les jeunes individus qui ont disparu, ceci à cause de leur plus grande sensibilité, les plus gros ou les plus âgés étant épargnés.

L'intérêt principal de cette étude a été la mise en évidence de l'importance des paramètres biotiques tel que la taille et le métabolisme des individus dans la variation de leur sensibilité et plus particulièrement dans le cas d'intoxication infralétale, lesquelles interviennent dans les cas de pollution non intentionnelle.

De même la variation de facteur abiotique tel que la température en agissant directement sur le métabolisme des invertébrés aura des conséquences non négligeables.

Aussi la recherche d'indices nouveaux, prenant en compte le plus grand nombre de paramètres possible doit être envisagée très sérieusement, si l'on veut estimer avec plus de précisions les risques de pollution par les résidus. L'utilisation dans ce domaine des CL50 et DL50 donnant trop souvent de fausses estimations et minimisant les risques encourus. 


\section{RÉFĖRENCES BIBLIOGRAPHIQUES}

ANDERSON P.D., WEBER L.J., 1975. Toxic response as a quantitative function of body size. Toxicol. Appl. Pharmacol., $33: 471$ 483.

BLISS C.I., 1936. The size factor in the action of arsenic upon silkworm larvae. J. Exp. Biol., 33 : 95-110.

BUIKEMA A.L., NIEDERLEHNER B.R., CAIRNS J., 1982. Biological monitoring. Part IV - Toxicity Testing. Water Res., 16: 239-262.

FINNEY D.E., 1971. Probit Analysis, 3 rd edn., Cambridge univer. Press, London, England., $333 \mathrm{p}$.

FISCHER R.A., YATES F., 1963. Statistical tables for biological, agricultural and medical research. Longman, $146 \mathrm{p}$.

FUKANO K.J., HOOPER F.F., 1958. Toxaphene as a selective fish poison. Prog. Fish. Cult., $20: 189-190$.

HEMPHILL J.E., 1954. Toxaphene as a fish toxin. Prog. Fish. Cult., 16: 41-42.

HYNES H.B.N., 1960. The Biology of polluted waters. Liverpool University Press.

LE BRAS S., 1987. Influence du lindane sur le métabolisme respiratoire d'Asellus aquaticus $\mathrm{L}$. Relation concentration-perturbation. Hydrobiologia, 148: 115-122.
PHILLIPS J.H., 1980. Quantitative aquatic biological indicators. Kenneth Mellanby Ed. Appl. Sc. Pub. 488 p.

STEPHENSON R.R., 1983. Effects of water hardness, water temperature, and size of test organism on the susceptibility of the freshwater shrimp, Gammarus pulex L., to toxicants. Bull. Environ. Contam. Toxicol., 31: $459-466$.

THYBAUD E., 1987. Recherches sur l'impact écotoxicologique du lindane et de la deltaméthrine sur divers niveaux d'organisation des écosystèmes limniques. Diplóme de Doctorat de Toxicologie, Université de Paris VII. 245 p.

THYBAUD E., LE BRAS S., 1988. Adsorption and elimination of lindane by Asellus aquaticus (Crustacea, Isopoda). Bull. Environ. Contam. Toxicol, $40: 731-735$.

THYBAUD E., LE BRAS S., COSSON R.P., 1987. Etude comparée de la sensibilité d'Asellus aquaticus L. (Crustacé, Isopde) vis-à-vis de quelques insecticides et de divers métaux lourds. Acta Oeco., Oeco Appl., 8: 355-361.

TUCKER R.K., LEITZKE J.S., 1979. Comparative toxicology of insecticides for vertebrate wildlife and fish. Pharmac. Ther., 6: 167 . 220. 